\title{
Natural candidates for superheavy dark matter in string and $M$ theory
}

\author{
Karim Benakli, ${ }^{1}$ John Ellis, ${ }^{2}$ and Dimitri V. Nanopoulos ${ }^{1,3}$ \\ ${ }^{1}$ Center for Theoretical Physics, Department of Physics, Texas A \& M University, College Station, Texas 77843-4242 \\ ${ }^{2}$ Theory Division, CERN, CH-1211 Geneva 23, Switzerland \\ ${ }^{3}$ Astroparticle Physics Group, HARC, Mitchell Campus, The Woodlands, Texas 77381 \\ and Chair of Theoretical Physics, Academy of Athens, 28 Panepistimiou Avenue, Athens GR-10679, Greece
}

(Received 15 April 1998; published 25 January 1999)

\begin{abstract}
We reconsider superheavy dark matter candidates in string and $M$ theory, in view of the possibility that inflation might generate superheavy particles with an abundance close to that required for a near-critical Universe. We argue that cryptons - stable or metastable bound states of matter in the hidden sector-are favored over other possible candidates in string or M theory, such as the Kaluza-Klein states associated with extra dimensions. We exhibit a specific string model that predicts cryptons as hidden-sector bound states weighing $\sim 10^{12} \mathrm{GeV}$, and discuss their astrophysical observability. [S0556-2821(98)04824-3]

PACS number(s): 95.35.+d, 11.25.Mj, 12.60.Jv
\end{abstract}

There is striking evidence from astronomical observations for the existence of dark matter. The rotational velocities of galaxies, the dynamics of galaxy clusters, and theories of structure formation suggest that most of the matter in the Universe is invisible and largely composed of nonbaryonic particles. Many candidates have been proposed as constituents of this particle dark matter. An upper bound on the self-annihilation cross section, based on unitarity, suggests that particles much heavier than $1 \mathrm{TeV} \sim \sqrt{M_{\mathrm{Pl}} \times T_{\mathrm{CMBR}}}$ that have been in thermal equilibrium would be left with such a large relic abundance that they would overclose the Universe [1]. This rule could be evaded if there was significant entropy generation after they went out of thermal equilibrium. This possibility was raised in connection with one class of superheavy dark matter candidates: cryptons [2], which are stable or metastable bound states of matter in a hidden sector of string theory. However, there was until recently little reason to expect that the abundance of cryptons or other superheavy relics would be such as to constitute most of the mass density of dark matter.

The question of the abundance of superheavy relics has recently been reexamined [3-5]. In particular, a gravitational mechanism was suggested $[4,5]$ whereby cosmological inflation may generate a desirable abundance of such massive and weakly interacting massive relic particles. Numerical analysis indicates that the process may be largely independent of the details of the models considered for most properties of the dark matter constituent, as well as of the details of the transition between the inflationary phase and the subsequent thermal radiation-dominated phase. In the light of this new proposal, it is interesting to reexamine the possibility that cryptons or other superheavy string relics may constitute an important part of the astrophysical dark matter.

We argue below that the combination of metastability and a desirable relic density are more likely for cryptons than for superheavy Kaluza-Klein states associated with the extra dimensions compactified at short distance scales in string or $\mathrm{M}$ theory. We also discuss the possible observability of cryptons via their high-energy decay products.

We first review some basic facts about superheavy dark matter and the new proposals $[4,5]$ for relic production dur- ing inflation. To be of interest in the Universe today, any such relic particle $X$ should have a lifetime at least of the order of the age of the Universe, $\tau_{X} \geqslant 10^{10} \mathrm{y}$, and there may be more stringent limits coming from searches for its decay products, that depend on its mass and decay modes [6]. One should also require that its energy density $\rho_{X}\left(t_{0}\right)$ at the present time $t_{0}$ does not overclose the universe, i.e., $\Omega_{X}$ $\equiv \rho_{X}\left(t_{0}\right) / \rho_{C}\left(t_{0}\right)<1$, where the critical density $\rho_{C}\left(t_{0}\right)$ $=3 H_{0}^{2} M_{\mathrm{Pl}}^{2} / 8 \pi$, with $H_{0}$ the Hubble expansion rate today.

If the particle $X$ was in thermal equilibrium with the primordial plasma, the expected number of particles today $n_{0}$ is inversely proportional to the self-annihilation cross section $\sigma_{A}$. If the latter is bounded above by $\mathcal{O}\left(1 / M_{X}^{2}\right)$, as suggested by unitarity in a finite number of partial waves, then one reaches the usual conclusion that dark matter particle masses are unlikely to exceed greatly the electroweak scale. However, the dark matter candidate might avoid this constraint if it has never reached local thermal equilibrium with the primordial plasma. This situation is realized if $n_{X}\left\langle\sigma_{A}|v|\right\rangle \lesssim H$, where $n_{X}$ is the (conserved) comoving number density and $|v|$ is the Møller speed for the dark matter particles $X$. Given the usual dependence of $\sigma_{A}$ on the mass and coupling of $X$, the absence of thermal equilibrium requires that (i) $X$ is weakly interacting and (ii) $M_{X}$ is of the order of the Hubble constant.

However, without the constraint of thermal equilibrium, how does one explain why the superheavy particle is naturally generated in the correct number to form the cold dark matter of the universe? This problem was addressed by the authors of Refs. [4,5]. They suggested that these particles might be created through the interaction of the vacuum with the gravitational field during the reheating period of the universe [7]. Such a process involves only the gravitational interactions of the particle, and thus is quite independent of the other (weak) interactions that it might have. This scenario leads to the following mass density of the particle $X$ created at time $t=t_{e}[4]$ :

$$
\Omega_{X} h^{2} \approx \Omega_{R} h^{2}\left(\frac{T_{\mathrm{RH}}}{T_{0}}\right) \frac{8 \pi}{3}\left(\frac{M_{X}}{M_{\mathrm{Pl}}}\right) \frac{n_{X}\left(t_{e}\right)}{M_{\mathrm{Pl}} H^{2}\left(t_{e}\right)},
$$


where $\Omega_{R} h^{2} \approx 4.31 \times 10^{-5}$ is the fraction of the critical energy density that is in radiation today, and $T_{\mathrm{RH}}$ is the reheating temperature.

The numerical analysis of Ref. [4] indicates that the correct magnitude for the abundance of the $X$ particle is obtained if its mass lies in the region $0.04 \leq M_{X} / H \lesssim 2$, where $H \sim 10^{13} \mathrm{GeV}$ is the Hubble expansion rate at the end of inflation, which is expected to be of the same order as the mass of the inflaton. For our purposes, we shall consider the range $10^{11} \mathrm{GeV} \lesssim M_{X} \lesssim 10^{14} \mathrm{GeV}$ to be favorable for superheavy dark matter, and consider next various candidates for the $X$ particle within the context of string and $\mathrm{M}$ theory.

String theories have historically been analyzed in the weak-coupling limit, where there is an observable sector containing the known gauge interactions and matter particles, and a hidden sector that is expected to become strongly interacting and may play a role in supersymmetry breaking. In addition to the states that are massless before this and subsequent stages of symmetry breaking, such string models also contain Kaluza-Klein excitations with masses related to the scales at which surplus dimensions are compactified. In the weak-coupling limit, all these states would have masses comparable to the Planck mass $M_{\mathrm{Pl}}$ $\sim 10^{19} \mathrm{GeV}$, beyond the range favored by $[4,5]$. However, the string mass estimate may be revised downwards in the strong-coupling limit described by $\mathrm{M}$ theory, requiring a revised discussion as provided below. We now discuss in more detail some specific string and $\mathrm{M}$ theory possibilities.

(i) Level-one heterotic-string models. These have been the most studied vacua of string theory. The possibility of building explicit models and carrying out detailed computations makes possible a precise analysis. A well-established prediction of this class of compactifications is the existence of light (massless at the string scale) states which are singlets under $\mathrm{SU}(3)_{c}$ and carry fractional electrical charges, that appear generically in the hidden gauge-group sector $[8,9]$. Such particles cannot be free, because the lightest of these particles would have to be stable and present in the Universe with a large abundance. There are very stringent upper limits on the abundance of such a fractionally charged relic, from successors of the Milliken experiments, which are many orders of magnitude below the critical density. However, theoretical expectations for their abundance on Earth are about ten orders of magnitude above these limits [10]. Thus the only viable string vacua are those where these charges are confined by a "hidden" group $G$, as in QCD. The integercharged lightest singlet bound states of such a hidden-sector group may be stable or metastable, providing the dark-matter candidates termed cryptons [11].

The confining group $G$ must be such that singlet bound states of $\mathrm{SU}(3) \times G$ have integer electric charges. For $G$ $=\Pi_{N} \mathrm{SU}(N) \times \Pi_{n} \mathrm{SO}(2 n)$, this condition states that $[9,11]$

$$
\sum_{N} \frac{i_{N}\left(N-i_{N}\right)}{2 N}+\sum_{n} \begin{cases}0 & \text { for } j_{n}=0 \\ 1 / 2 & \text { for } j_{n}=2 \\ n / 8 & \text { for } j_{n}=1\end{cases}
$$

must be a nonvanishing integer, where for every $N, i_{N}$ is some integer between 0 and $N-1$. Thus the electric charge of a state transforming in the representation $N$ or $\bar{N}$ of $\mathrm{SU}(N)$ and/or $2 n$ of $\mathrm{SO}(2 n)$ must be

$$
q= \pm \sum_{N} \frac{i_{N}}{N}+\sum_{n} \frac{j_{n}}{2} \quad \bmod 1
$$

with \pm corresponding to representations $N$ or $\bar{N}$.

The case where $G$ is a product of semisimple factors presents the advantage, compared to a large unique semisimple group, of generally giving rise to a smaller number of fractionally charged states that have to be included in the running of the supersymmetric standard-model gauge couplings $[12,13]$. Note also that, because of these states, the $G$ gauge sector is not completely "hidden." This may even be advantageous, if supersymmetry is broken when the coupling of $G$ becomes strong, and an $F$ term is generated. This supersymmetry breaking would be mediated to the observable sector not only by gravitational interactions involving the graviton supermultiplet, but also through the usual standard-model gauge interactions via the supermultiplets of fractionally charged states [13].

(ii) Higher-level string models. This class of constructions is largely motivated by the need to accommodate adjoint Higgs representations in grand unified theories (GUTs) other than flipped $\mathrm{SU}(5)$. In so doing, they usually also lead to new exotic matter. In the specific case of standard $\mathrm{SU}(5)$ realized at level 2, there were found representations transforming as $(8,1,0),(1,3,0)$, and $(1,1,0)$ of $\mathrm{SU}(3) \times \mathrm{SU}(2) \times \mathrm{U}(1)$ [14]. These particles were also found to have no superpotential, and hence should appear below the string scale. It was suggested in Ref. [15] that these states might have masses of the order of $10^{13} \mathrm{GeV}$, so as to resolve apparent discrepancy between the unification and string scales. In such a scenario, the singlet $(1,1,0)$ state could be a dark matter candidate. In common with the levelone constructions, these models also have a hidden sector where supersymmetry breaking might originate, which might also provide a stable bound state as discussed above.

(iii) $M$ theory on $S^{1} / Z_{2}$. The first phenomenological studies of $M$ theory have raised the possibility that six of the original eleven dimensions might be compactified at an energy scale comparable to the conventional supersymmetric GUT scale, in the range of $10^{16}$ to $10^{17} \mathrm{GeV}$, leaving an effectively five-dimensional low-energy theory. The fifth dimension would subsequently be compactified down to four dimensions, on an $S^{1} / Z_{2}$ segment with a size $\rho$ that might be of order $10^{-13}$ to $10^{-15} \mathrm{GeV}^{-1}$ [16]. "Hexon" KaluzaKlein states associated with the $11 \rightarrow 5$ compactification are likely to be too heavy to have been produced copiously via the mechanism of Refs. [4,5], but massive states associated with the $5 \rightarrow 4$ compactification might well have masses in the favored range [17]. We term such Kaluza-Klein states "pentons." In this type of M-theory scenario, the effective field theory at energies below about $10^{16} \mathrm{GeV}$ is $N=2 \mathrm{su}-$ pergravity, which in the specific case of $\mathrm{M}$ theory compactified on a Calabi-Yau manifold has has been shown [18] to contain $h_{1,1}-1$ vector hypermultiplets and $h_{2,1}+1$ scalar hy- 
TABLE I. The spectrum of hidden matter fields that are massless at the string scale in the revamped flipped SU(5) model. We display the quantum numbers under the hidden gauge group $\mathrm{SO}(10) \times \mathrm{SO}(6) \times \mathrm{U}(1)^{4}$, and subscripts indicate the electric charges.

\begin{tabular}{lc}
\hline \hline$\Delta_{1}^{0}\left(0,1,6,0,-\frac{1}{2}, \frac{1}{2}, 0\right)$ & $\Delta_{2}^{0}\left(0,1,6,-\frac{1}{2}, 0, \frac{1}{2}, 0\right)$ \\
$\Delta_{3}^{0}\left(0,1,6,-\frac{1}{2},-\frac{1}{2}, 0, \frac{1}{2}\right)$ & $\Delta_{4}^{0}\left(0,1,6,0,-\frac{1}{2}, \frac{1}{2}, 0\right)$ \\
$\Delta_{5}^{0}\left(0,1,6, \frac{1}{2}, 0,-\frac{1}{2}, 0\right)$ & \\
$T_{1}^{0}\left(10,1,0,-\frac{1}{2}, \frac{1}{2}, 0\right)$ & $T_{2}^{0}\left(10,1,-\frac{1}{2}, 0, \frac{1}{2}, 0\right)$ \\
$T_{3}^{0}\left(10,1,-\frac{1}{2},-\frac{1}{2}, 0, \frac{1}{2}\right)$ & $T_{4}^{0}\left(10,1,0, \frac{1}{2},-\frac{1}{2}, 0\right)$ \\
$T_{5}^{0}\left(10,1,-\frac{1}{2}, 0, \frac{1}{2}, 0\right)$ & \\
& \\
$\widetilde{F}_{1}^{+(1 / 2)}\left(1,4,-\frac{1}{4}, \frac{1}{4},-\frac{1}{4}, \frac{1}{2}\right)$ & $\widetilde{F}^{+(1 / 2)}{ }_{2}\left(1,4,-\frac{1}{4}, \frac{1}{4},-\frac{1}{4},-\frac{1}{2}\right)$ \\
$\widetilde{F}_{3}^{-(1 / 2)}\left(1,4, \frac{1}{4}, \frac{1}{4},-\frac{1}{4}, \frac{1}{2}\right)$ & $\widetilde{F}_{4}^{+(1 / 2)}\left(1,4, \frac{1}{4},-\frac{1}{4},-\frac{1}{4}, 6-\frac{1}{2}\right)$ \\
$\widetilde{F}_{5}^{+(1 / 2)}\left(1,4,-\frac{1}{4}, \frac{3}{4},-\frac{1}{4}, 0\right)$ & $\widetilde{F}_{6}^{+(1 / 2)}\left(1,4,-\frac{1}{4}, \frac{1}{4},-\frac{1}{4},-\frac{1}{2}\right)$ \\
$\tilde{F}_{1}^{-(1 / 2)}\left(1,4,-\frac{1}{4}, \frac{1}{4}, \frac{1}{4}, \frac{1}{2}\right)$ & $\tilde{F}_{2}^{-(1 / 2)}\left(1,4,-\frac{1}{4}, \frac{1}{4}, \frac{1}{4},-\frac{1}{2}\right)$ \\
$\tilde{F}_{3}^{+(1 / 2)}\left(1,4,-\frac{1}{4},-\frac{1}{4}, \frac{1}{4},-\frac{1}{2}\right)$ & $\tilde{F}_{4}^{-(1 / 2)}\left(1,4,-\frac{1}{4}, \frac{1}{4}, \frac{1}{4},-\frac{1}{2}\right)$ \\
$\tilde{\tilde{F}}_{5}^{-(1 / 2)}\left(1,4,-\frac{3}{4}, \frac{1}{4},-\frac{1}{4}, 0\right)$ & $\tilde{F}_{6}^{-(1 / 2)}\left(1,4, \frac{1}{4},-\frac{1}{4}, \frac{1}{4},-\frac{1}{2}\right)$ \\
\hline \hline
\end{tabular}

permultiplets. None of these carry conventional gauge interactions, which are realized on the walls.

The pentons, i.e., the massive Kaluza-Klein bulk excitations of this particular five-dimensional supergravity theory, therefore do not carry any new conserved quantum number, in contrast to generic "pyrgons" [19]. These states are free to decay into fields living on one of the walls of universe, and the most stable pentons would be those with the smallest couplings to boundary fields. We expect these to be of the order of the gravitational constant. Thus their lifetimes should be of order $\rho^{3} / G_{N}$, which is much too short to constitute the dark matter in the Universe today.

A more likely candidate might emerge among the states living on the wall at the (hidden) opposite wall of the fivedimensional bulk from the conventional observable sector. As in the previous discussion of weakly coupled string models, one would expect that the mass of the lightest crypton bound state would have its origin in non-perturbative strongcoupling effects, which might well occur at the favored scale of order $10^{13} \mathrm{GeV}[4,5]$. Similarly to protons on our (observable) wall, there would in general be some flavor symmetry of the hidden world which allows such a bound state to remain metastable, providing us with a candidate for the dark matter particle $X$.

We now review an explicit example of a string model whose hidden sector contains such metastable crypton bound states. This model was originally constructed in the weak-coupling limit [20], but we expect that it may be elevated to an authentic M-theory model in the strong-coupling limit. This model has the gauge group $\mathrm{SU}(5) \times \mathrm{U}(1) \times \mathrm{U}(1)^{4} \times \mathrm{SO}(10) \times \mathrm{SU}(4)$, with the latter two factors yielding strong hidden-sector interactions. The Table I lists the matter content of this hidden sector.

Analysis of the calculable superpotential in this model shows that most of these fields acquire heavy masses just below the string scale from couplings with singlet fields that acquire vacuum expectation values to cancel the $D$ term of the anomalous $\mathrm{U}(1)$. The only light states that survive to have lower masses are the $T_{3}, \Delta_{3}, \widetilde{F}_{3,5}$, and $\tilde{\bar{F}}_{3,5}$. Analysis of the renormalization-group $\beta$ functions of $\mathrm{SO}(10)$ and $\mathrm{SO}(6)$ suggest that their confinement scales might lie at $\Lambda_{10} \sim 10^{14-15} \mathrm{GeV}$ for $\mathrm{SO}(10)$ and $\Lambda_{4} \sim 10^{11-12} \mathrm{GeV}$ for $\mathrm{SU}(4)$. This indicates that the states in the SU(4) representations $\Delta_{3}, \widetilde{F}_{3,5}$, and $\tilde{F}_{3,5}$ will form the lightest bound states.

In addition to meson and baryon bound states as in QCD, one expects quadrilinear tetron bound states specific to SU(4) [2]. The mesons comprise $T_{i} T_{j}, \Delta_{i} \Delta_{j}$, and $\widetilde{F}_{i} \tilde{F}_{j}$ bound states, which are all short-lived, as they decay through order $N=3,4$ or 6 nonrenormalizable operators. The baryons have the constituents $\widetilde{F}_{i} \widetilde{F}_{j} \Delta_{k}$ and $\tilde{\bar{F}}_{i} \tilde{\bar{F}}_{j} \Delta_{k}$ are also short lived. Finally, there are tetrons composed of four $\widetilde{F}_{i} \mathrm{~s}$, of which the lightest have the forms $\widetilde{F}_{i} \widetilde{F}_{j} \widetilde{F}_{k} \widetilde{F}_{l}$ and $\tilde{\bar{F}}_{i} \tilde{\tilde{F}}_{j} \tilde{\bar{F}}_{k} \tilde{\tilde{F}}_{l}$, where $i, j, k, l=3,5$. As in the case of QCD pions, one may expect the charged states [21] to be slightly heavier than the neutral ones, due to electromagnetic energy mass splitting. No nonrenormalizable interaction capable of enabling this lightest bound state to decay has been found in a search up to eighth order. We therefore consider that this lightest neutral tetron is a perfect candidate for a superheavy dark matter particle. A rough lower bound on the lifetime of this lightest tetron is of the order

$$
\tau_{X} \sim \frac{1}{M_{X}}\left(\frac{m_{k}}{M_{X}}\right)^{10},
$$

which is very sensitive to $M_{X}$ and the scale $m_{k}$ of suppression of the nonrenormalizable terms. For $m_{k} \sim 10^{17}$ $10^{18} \mathrm{GeV}$, and a tetron mass $M_{X} \sim 10^{12} \mathrm{GeV}$, we find that $\tau_{X}>10^{7}-10^{17} \mathrm{yr}$. This is a lower bound, and the actual lifetime may well be considerably longer if the leading decay interaction is of significantly higher order.

If cryptons are unstable, their decays might explain the ultrahigh-energy cosmic ray events [22] observed beyond the Greisen-Zatsepin-Kuzmin (GZK) cutoff energy [23]. Explanations of these events as decays of superheavy relic particle has been considered by many authors [5,24,25]. If the $X$ particles form the same proportions of dark matter in the galaxy and in intergalactic space, it was argued in Ref. [25] that the observed extensive air showers are mainly due to gamma rays and nucleons produced by $X$ particle decays in the halo of our galaxy. This explains the absence of the GZK cutoff, and one easily avoids constraints on the cascade radiation.

The observed fluxes constrain $\Omega_{X} / \tau_{X}$. For $\tau_{X} \sim 10^{10}$ years, the density of $X$ particles is $\Omega_{X} \sim 10^{-21}$. For the range $\Omega_{X} \sim 1$ of interest to us, we could match the observed fluxes if $10^{15}$ years $\lesssim \tau_{X} \lesssim 10^{22}$ years $[6,5]$. In contrast to other superheavy particles that decay via nonperturbative gauge (instanton) or quantum-gravity (wormhole) interactions, our candidate has the advantage that decays via nonrenormalizable operators that might be explicitly computed in a perturbative framework. The desired value might be attainable in the explicit model discussed above if, for example, $m_{k}$ $\sim 10^{17} \mathrm{GeV}$ and the first decay interaction is of order $N$ 
$=9$ or higher. As discussed in Ref. [5], the ultrahigh-energy cosmic rays produced by the crypton decay might present a signature that distinguish them from other possible sources: the cosmic-ray spectrum should have a new cutoff at an energy $\lesssim M_{X}$.

To conclude, we have reevaluated possible candidates for superheavy dark matter in string or M theory, in the light of new production estimates $[4,5]$. In particular, we have revisited the proposal that cryptons may be an important component of dark matter. These particles have been shown to exist in a particular model with the desired mass, very weak inter- actions, and long lifetimes. The possibility that they are generated by gravitational interactions with the vacuum [4,5] may answer the long-standing question of why their abundance should lie in the interesting range $\Omega \sim 0.1$ to $\sim 0.9$. We have also discussed other dark-matter candidates in the context of string and $\mathrm{M}$ theory, such as hexons and pentons, but these do not have the desired mass and/or metastability, at least in the models studied so far.

The work of K.B. (D.V.N.) was supported by U.S. DOE Grant No. DE-FG03-95-ER-40917 (DE-FG05-91-GR40633).
[1] K. Griest and M. Kamionkowski, Phys. Rev. Lett. 64, 615 (1990).

[2] J. Ellis, J. L. Lopez, and D. V. Nanopoulos, Phys. Lett. B 247, 257 (1990).

[3] E. W. Kolb, A. D. Linde, and A. Riotto, Phys. Rev. Lett. 77, 4290 (1996); B. R. Greene, T. Prokopec, and T. G. Roos, Phys. Rev. D 56, 6484 (1997); E. W. Kolb, A. Riotto, and I. I. Tkachev, Phys. Lett. B 423, 348 (1998).

[4] D. J. H. Chung, E. W. Kolb, and A. Riotto, Phys. Rev. D 59, 023501 (1999).

[5] V. Kuzmin and I. Tkachev, hep-ph/9802304.

[6] J. Ellis, G. B. Gelmini, J. L. Lopez, D. V. Nanopoulos, and S. Sarkar, Nucl. Phys. B373, 399 (1992).

[7] See, for example, N. D. Birrell and P. C. W. Davies, Quantum Fields in Curved Space (Cambridge University Press, Cambridge, England, 1982).

[8] X. Wen and E. Witten, Nucl. Phys. B261, 651 (1985).

[9] A. Schellekens, Phys. Lett. B B237, 363 (1990).

[10] G. Athanasiu, J. Atick, M. Dine, and W. Fischler, Phys. Lett. B 214, 55 (1988).

[11] J. Ellis, J. L. Lopez, and D. V. Nanopoulos, Phys. Lett. B 245, 375 (1990).

[12] D. Bailin and A. Love, Mod. Phys. Lett. A 7, 1485 (1992).

[13] I. Antoniadis and K. Benakli, Phys. Lett. B 295, 219 (1992); 407, 449E (1997).
[14] G. Aldazabal, A. Font, L. E. Ibañez, and A. M. Uranga, Nucl. Phys. B452, 3 (1995).

[15] C. Bachas, C. Fabre, and T. Yanagida, Phys. Lett. B 370, 49 (1996).

[16] P. Horava and E. Witten, Nucl. Phys. B460, 506 (1996); E. Witten, ibid. B471, 135 (1996).

[17] It could even be that inflation is associated with the $5 \rightarrow 4$ transition, but we do not pursue this possibility here.

[18] I. Antoniadis, S. Ferrara, and T. R. Taylor, Nucl. Phys. B460, 489 (1996).

[19] E. W. Kolb and R. Slansky, Phys. Lett. 135B, 378 (1984).

[20] I. Antoniadis, J. Ellis, J. Hagelin, and D. V. Nanopoulos, Phys. Lett. B B231, 65 (1989).

[21] A. De Rujula, S. L. Glashow, and U. Sarid, Nucl. Phys. B333, 173 (1992).

[22] N. Hayashida et al., Phys. Rev. Lett. 73, 3491 (1994); D. J. Bird et al., Astrophys. J. 424, 491 (1994); 441, 144 (1995); T. A. Egorov et al., in Proceedings of the Tokyo Workshop on Techniques for the Study of Extremely High Energy Cosmic Rays, edited by M. Nagano, ICRR, University of Tokyo, 1993 (unpublished).

[23] K. Greisen, Phys. Rev. Lett. 16, 748 (1966); G. T. Zatsepin and V. A. Kuzmin, Pis'ma Zh. Eksp. Teor. Fiz. 4, 114 (1966).

[24] V. A. Kuzmin and V. A. Rubakov, Phys. At. Nucl. 61, 1028 (1998).

[25] V. Berezinsky, M. Kachelriess, and A. Vilenkin, Phys. Rev. Lett. 79, 4302 (1997). 